\title{
A New Finite Element Combination Method to Solve the Crack Cylinder of the Saint-Venant's Torsion Problem
}

\author{
Xinyan Tang \\ Department of Mechanical Engineering, Engineering College, Nanjing Agricultural University, Nanjing Jiangsu 210031 \\ China \\ e-mail: tangxiyan@njau.edu.cn
}

\begin{abstract}
Based on the Saint-Venant's problem, combine the Crack3D code and Ansys software, a new finite element combination method to calculate the Saint-Venant's torsion problem including crack is proposed. In order to illustrate its application, the numerical example for a Saint-Venant's torsion problem with crack are given and the numerical results are satisfactory.
\end{abstract}

Keywords-finite element combination method; crack cylinder; Saint-Venant's torsion problem

\section{INTRODUCTION}

This paper combined the Crack3D FEA code [1], developed by Drs Jianzheng Zuo, Xiaomin Deng and Michael A. Sutton., in the Department of Mechanical Engineering at the University of South Carolina, SC, U.S.A. and general software Ansys to get the combination finite element method to calculate the Saint-Venant's torsion problem including crack. In the example, some numerical results such as the stress intensity factor KIII, torsion rigidity $\mathrm{D}$, shearing stresses $\left(\tau_{z x}, \tau_{z y}\right)$ etc. are obtained. From the numerical results, it appears the combination finite element method is successful and quite powerful.

\section{SAINT-VENANT'S TORSION PROBLEM}

According to the Saint-Venant's torsion problem[2], in rectangular coordinates $(x, y, z)$, the displacement components of the cylinder applied by torsion moment $M_{k}$ can be expressed as follows:

$$
\left\{\begin{array}{l}
u_{x}=-\alpha z y \\
u_{y}=\alpha z x \\
u_{z}=\alpha \varphi(x, y)
\end{array}\right.
$$

where $\alpha$ is the twisting angle per unit length of the cylinder, i.e. twisting ratio, which can be determined by the relative angle of rotation between two different cross-sections. If the torsion cylinder region is $\Omega$, letting $\varphi(x, y)$ be a plane harmonic function in $\Omega$, the torsion rigidity $D$ is determined using the following area integral

$$
\begin{aligned}
& D=\frac{M_{k}}{\alpha}=\mu \int_{\Omega}\left(x \frac{\partial \phi}{\partial y}-y \frac{\partial \phi}{\partial x}+x^{2}+y^{2}\right) d \Omega \text { (2) } \\
& \mu=\frac{E}{2(1+v)} \text { is the shearing modulus. } \\
& \text { in which } \\
& \text { The corresponding torsion shearing stresses } \\
& \left(\tau_{z x}, \tau_{z y}\right) \text { on } \Omega \text { are given by }
\end{aligned}
$$

$$
\left\{\begin{array}{l}
\tau_{z x}(x, y)=\alpha \mu\left[\frac{\partial \phi}{\partial x}-y\right] \\
\tau_{x y}(x, y)=\alpha \mu\left[\frac{\partial \phi}{\partial y}+x\right]
\end{array}\right.
$$

\section{THE COMBINATION FINITE ELEMENT METHOD TO SOLVE SAINT-VENANT'S TORSION PROBLEM}

To the author's knowledge, the boundary element method [3] has been used extensively to solve the Saint-Venant's torsion problem in elasticity. In solid mechanics, the finite element method[4] (FEM) is a well known, versatile, flexible numerical method that is being used extensively in almost all fields of science and engineering. Since it does not appear that the FEM has been used to computationally solve the Saint-Venant's torsion problem, one of the goals of this paper is to outline a combination of theory and FEM analyses to solve an equivalent Saint-Venant's torsion cylinder. Here the CRACK3D code with ANSYS software finite element method will be used to solve the general torsion cylinder in order to get the relative numerical results on the boundary $\Gamma$ equivalent to the Saint-Venant's torsion solution. After substituting these results into the boundary element discretion formula derived from Saint-Venant's torsion theory, the torsion numerical solution for the equivalent Saint-Venant's cylinder torsion problem is obtained.

In order to explain the combination method used here, the essential patterns to solve the equivalent problem are outlined below.

Step 1. Establish the geometric model in an Ansys Preprocessor. Mesh the geometric model using 8-noded brick element. 
Step 2. Using following GUI operations[5]to obtain the NODES.DAT and ELEMS.DAT files.

Main menu/Preprocessor/Create/Nodes//Write Node File.

Main menu/Preprocessor/Create/Elements/Write Elem File.

Step 3. Insert NODES.DAT file and ELEMS.DAT file into MESH3D interface and execute the Pre-Interface Code MESH3D to generate the data file CRACK3D.MSH.

Step 4. Using the NODES.DAT file and ELEMS.DAT file, establish the controlling data file CRACK3D.DAT

Step 5. Insert the data files CRACK3D.MSH and CRACK3D.DAT into the CRACK3D interface and execute the main code CRACK3D to obtain the numerical results of CRACK3D.ANS and CRACK3D.RES.

Step 6. Insert the binary neutral data file CRACK3D.ANS into POST3D interface and execute the Post-Interface code POST3D to transform the obtained result data to ANSYS Post-Processor data format CRACK3D.RST.

Step 7. Read the data file CRACK3D.RST in ANSYS Post-Processor. Then the ANSYS Post-Processor can be used for displaying displacement, stress and strain fields.

Step 8. From ANSYS Post-Processor or the CRACK3D.RST. Take the node's displacements $u_{z}$ on the boundary $\Gamma$ of mid cross-section $\Omega$ and use the theoretical formula (1) to determine the boundary values of torsion function $\varphi(x, y)$ at boundary $\Gamma$ as equation (4). Use Green's formula to express the area integral in equation (2) as a line integral as equation (5) along the boundary $\Gamma$. Finally, discretize the boundary $\Gamma$ by boundary elements and calculate the dimensionless parameter $D / \mu a^{4}$ of the twisting rigidity $D$ for the equivalent cylinder $\Omega$ numerically for comparison with the theoretical result:

$$
\begin{gathered}
\phi(x, y)=\frac{u_{z}(x, y)}{\alpha} \\
\frac{D}{\mu}=\int_{\Omega}\left(x \frac{\partial \phi}{\partial y}-y \frac{\partial \phi}{\partial x}+x^{2}+y^{2}\right) d \Omega=\int_{\Gamma} \frac{1}{3}\left(x^{3} n_{x}+y^{3} n_{y}\right) d \Gamma+\int_{\Gamma} \phi\left(x n_{y}-y n_{x}\right. \\
=\int_{\Gamma} \frac{1}{3}\left(x^{3} n_{x}+y^{3} n_{y}\right) d \Gamma+\frac{1}{\alpha} \int_{\Gamma} u_{z}\left(x n_{y}-y n_{x}\right) d \Gamma \\
\frac{D}{\mu a^{4}}
\end{gathered}
$$

Step 9. From ANSYS Post-Processor or the CRACK3D.RST to take the stresses, strains and all the needed results. After that using the two maximum shearing stresses $\tau\left(\tau_{1}, \tau_{2}\right)$ at two points on boundary

$\Gamma$ of the square cross-section $\Omega$, to determine following two dimensionless parameters and compare with the theoretical results:

$$
\frac{\tau_{1}}{\alpha \mu a}, \quad \frac{\tau_{2}}{\alpha \mu a}
$$

Step 10. From ANSYS Post-Processor or the CRACK3D.RST, take the node's reactions and determine the torsion moment $M_{k}$. Using the maximum shearing stress $\tau_{\max }=\tau_{1}\left(\right.$ or $\left.\tau_{2}\right)$ at the boundary $\Gamma$ of the cross-section $\Omega$, calculate the following dimensionless parameter and compare with the theoretical result:

$$
\frac{M_{k}}{\tau_{\max } a^{3}}
$$

Step 11. In the case of the cross-section $\Omega$ with an edge crack, obtain the $C O D$ of the crack and determine the III- mode stress intensity factor at the crack tip by following formula[ 6 ]:

$$
K_{I I I}=\frac{\mu}{2} \lim _{r \rightarrow 0} \frac{C O D}{\sqrt{2 \pi r}}
$$

\section{NUMERICAL RESULTS OF SAINT-VENANT'S TORSION PROBLEM WITH CRACK}

We consider a equivalent Saint-Venant's torsion problem on a general cylinder $(20 \times 20 \times 60 \mathrm{~mm})$ with a square cross-section $\Omega$, and in the cross-section $\Omega$ contains an edge crack. The distance between top cutting section $\Omega_{t}$ and bottom cutting section $\Omega_{b}$ is taken as $l=30 \mathrm{~mm}$. The material constants are: Young's modulus $E=100 G P a$, Poisson's ratio $v=0.3$, initial yield stress $\sigma_{y}=100 \mathrm{MPa}$

Using the 8-node brick element model, the uniform element mesh is automatically divided by ANSYS software for this cylinder, where the element edge defngth is taken as $2.5 \mathrm{~mm}$.

The lower end section of the general cylinder is fixed by 6 nodes to prevent rotation ( node's numbers: $627,1626,1126,6,1$ and 2 ), but the end is allowed to extend in the $z$ direction. The upper end section is acted upon by a specified displacement at 5 nodes (646,1670,1146,146 and 50 ) with displacement loads

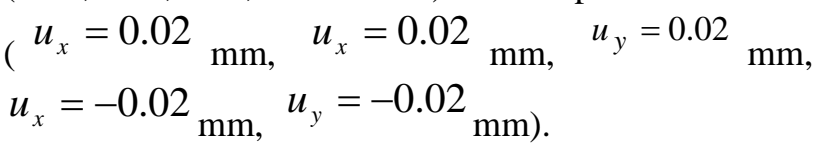




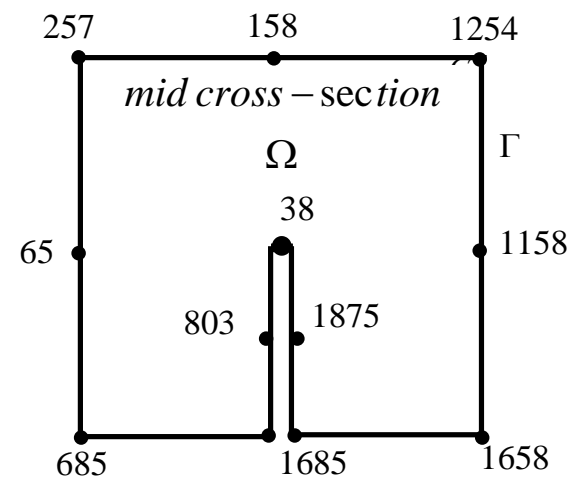

Fig. 1 The Boundary $\Gamma$ OF THE Mid Cross-SeCtion $\Omega$ With CRACK

The above torsion cracked problem is solved by CRACK3D code with ANSYS software, and the obtained results $\frac{D}{\mu a^{4}}, \frac{\tau}{\alpha \mu a}, \frac{M_{k}}{\tau a^{3}}$,and $K_{I I I}$ are given in table 1.

TABLE I THE RESULTS OBTAINED FROM CRACK3D CODE WITH ANSYS SOFTWARE CALCULATIONS ABOUT

$$
\frac{D}{\mu a^{4}} \frac{\tau}{\alpha \mu a} \frac{M_{k}}{\tau a^{3}} K_{I I I}
$$

\begin{tabular}{|c|c|}
\hline & $\begin{array}{c}\text { CRACK3D code with } \\
\text { ANSYS software }\end{array}$ \\
\hline$\frac{D}{\mu a^{4}}$ & 1.1051 \\
\hline (dimensionless) & \\
\hline$\frac{\alpha \mu a}{2}$ & 1.0783 \\
\hline
\end{tabular}

\begin{tabular}{|c|c|}
\hline (dimensionless) & \\
\hline$\frac{M_{k}}{\tau a^{3}}$ & 1.1849 \\
(dimensionless) & \\
\hline$K_{I I I}(N / m m 3 / 2)$ & 11.6780 \\
\hline
\end{tabular}

\section{CONCLUSIONS}

As shown in the tabular results given above, the results coincide well with the known theoretical results which indicate the combination finite element method used here appears to be a good approximation method to solve actual torsion problems.

\section{ACKNOWLEDGMENT}

The academic support is provided by the Department of Mechanical Engineering at the University of South Carolina in Columbia, SC. USA, including the Crack3D FEA Code software, hardware and office space, is gratefully acknowledged. The authors also appreciate Dr. Jianzheng Zuo and Mr. Hui Yang for helpful to run Crack3D and other software.

\section{REFERENCES}

[1] Jianzheng Zuo, Xiaomin Deng and Michael A. Sutton. User's Manual and Verifications in CRACK3D version 4.50, University of South Carolina, 2007.

[2] Muskhelishvili, Some Basic Problems of the Mathematical Theory of Elasticity, Netherlands, 1953.

[3] Brebbia,C.A., Boundary Element Techniques, Springger-Verlag, Berlin,1984.

[4] Zienkiewicz O.P., The Finite Element Method in Engineering Science, London,1971.

[5] Zhang LeLe, Ansys applied Course, Beijing, 2006.( in Chinese)

[6] Sun Xunfang, Fracture Mechanics Teaching Materials, Zhengzhou, 1980.(in Chinese) 